\title{
Evolution of grain grading and characteristics in repeatedly reconstituted assemblages subject to one-dimensional compression
}

\author{
W. M. YAN* and Y. SHI*
}

\begin{abstract}
One-dimensional compression tests were carried out on repeatedly reconstituted assemblages that were uniformly graded prior to the first compression. Four stress levels were adapted to observe the interplay among grain shapes and grading, overburden stress and the number of remouldings. It was found that a stable limiting grading can be obtained after several loadings on repeatedly remoulded specimens. The position of this limiting grading is a function of the stress level. The evolution of grain shape with remoulding was found to depend on the magnitude of the applied stress. The grading with a fractal dimension of 2.65 can only be achieved by loading the repeatedly reconstituted specimen at an elevated stress. Stable shape characteristics of the grains were observed at the fractal grading. The amount of breakage of an assemblage is described by the relative breakage $B_{\mathrm{r}}$ and the evolution of $B_{\mathrm{r}}$ of the virgin compressed and repeatedly compressed specimens with stress is discussed.
\end{abstract}

KEYWORDS: fractals; laboratory tests; particle crushing/crushability; repeated loading; sands

ICE Publishing: all rights reserved

\section{NOTATION}

$\begin{array}{ll}\text { AR } & \text { aspect ratio } \\ B_{\mathrm{r}} & \text { relative breakage } \\ C & \text { convexity } \\ D & \text { fractal dimension } \\ S & \text { sphericity } \\ \sigma & \text { vertical stress } \\ \text { NCL } & \text { normal compression line }\end{array}$

\section{INTRODUCTION}

Granular soils have been found to be crushable when subjected to sufficiently high stress or strain, which results in grading changes (e.g. Yasufuku \& Hyde, 1995; Lade \& Yamamuro, 1996; Leung et al., 1996; Nakata et al., 2001a, 2001 b; Coop et al., 2004; Miao \& Airey, 2013). The concept of fractal grading was proposed to describe the grain size distribution of a soil after substantial crushing (McDowell et al., 1996). In many cases, a fractal dimension of about $2 \cdot 5-2 \cdot 6$ is found to be applicable to describe the ultimate fractal grading of an assemblage of grains (McDowell \& Bolton, 1998). However, experimental data also show that the ultimate grading is sensitive to the stress level and initial grading of the grains, and is not always fractal (Coop et al., 2004; Miao \& Airey, 2013; Zhang \& Baudet, 2013).

Reconstituting a specimen using grains resulting from a previously compressed specimen creates an assemblage having the same grain size distribution as the previous one yet different packing and grain contacts. The grains are expected to crush further when this reconstituted specimen is subjected to another cycle of loading. However, some points remain unclear. Firstly, will this remoulding and repeated loading yield a limiting grading? Secondly, if there

Manuscript received 15 May 2014; first decision 27 June 2014; accepted 1 August 2014.

Published online at www.geotechniqueletters.com on 3 September 2014.

*Department of Civil Engineering, The University of Hong Kong, Pokfulam, Hong Kong, China exists a limiting grading, is it fractal (self-similarity)? This paper presents a systematic study to shed light on the above questions using one-dimensional (1D) compression tests on crushable grains. The evolution of grain grading and grain shape parameters with reconstitution and repeated loading is discussed.

\section{MATERIALS AND TEST PROCEDURES}

The material tested was an aquarium-use coral sand ( specific gravity $=2 \cdot 78$ ) with calcareous origins as verified by x-ray diffraction tests. As shown in Fig. 1, the grains were porous and generally elongated. The grains were first oven-dried at $105^{\circ} \mathrm{C}$ for $24 \mathrm{~h}$ prior to mechanical sieving to screen out the $1 \cdot 18-2 \cdot 00 \mathrm{~mm}$ grains (here called parent grains) which were then used to prepare uniformly graded assemblages. One-dimensional compression tests were performed at medium to high stresses. Before the test, QicPic optical analysis was performed on the grains in each specimen to obtain the shape characteristics (including aspect ratio, sphericity and convexity) of the grains. Aspect ratio (AR) indicates the sense of elongation of a grain while sphericity $(S)$ represents the degree of circularity of a grain. Convexity $(C)$ describes the compactness of a grain where a convex polygon (grain) gives $C=1$. Details of the QicPic analysis, including the operational procedures, can be found elsewhere (Altuhafi \& Coop, 2011). The air pluviation method was used to rain the grains into a stainless steel cylindrical container (height=diameter $=40 \mathrm{~mm}$ ) in a loose state. The inner surface of the container was coated with Teflon to reduce wall friction. Four series of tests were carried out, corresponding to vertical pressures of 4, 8, 18 and $36 \mathrm{MPa}$. In this study, the 4, 8 and $18 \mathrm{MPa}$ compression tests were conducted in a conventional oedometer apparatus while the $36 \mathrm{MPa}$ compression test was conducted using a MTS (model 815) loading machine. Axial displacement of the specimen was monitored to resolutions of $0.001 \mathrm{~mm}$ (oedometer) and $0.005 \mathrm{~mm}$ (MTS machine). The initial void ratio of a specimen prior to compression in each test was calculated 


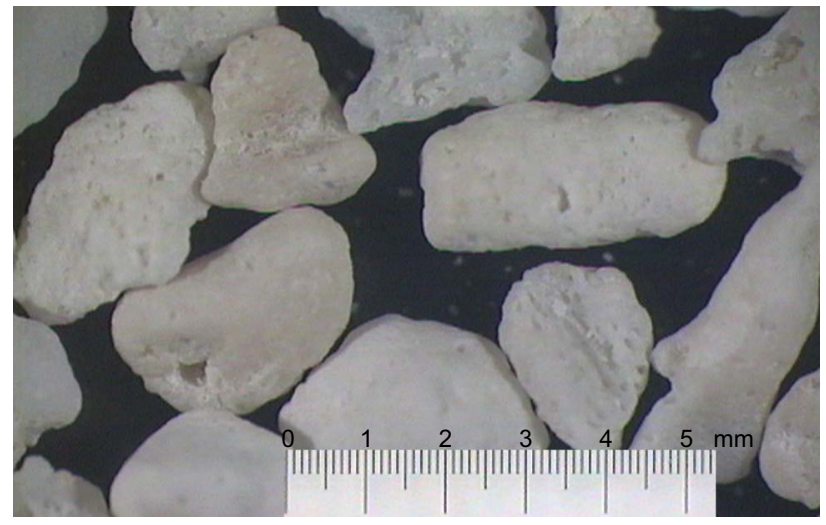

Fig. 1. The tested materials - porous coral sands with calcareous origins

from the mass of specimen (to a resolution of $0 \cdot 001 \mathrm{~g}$ ) and its dimensions, which were measured using high-precision callipers with a resolution of $0.01 \mathrm{~mm}$.

A specimen was first loaded to the target overburden stress in stages before being fully unloaded. Then, all the grains were taken out from the container for QicPic analyses and mechanical sieving was used to reveal grain shape parameters and size distributions. The grains (likely crushed after the loading-unloading cycle) were then used to reconstitute a new specimen, again to a loose state. A second loading-unloading cycle was then applied to this reconstituted specimen to the same target pressure. The process was repeated a few times until the change in grain grading became negligibly small. The tests were labelled according to target vertical stress and the number of reconstitutions: for example, test $8 \_0$ and test $8 \_5$, respectively, indicate the initial and the fifth reconstituted specimen subject to a vertical stress of $8 \mathrm{MPa}$.

\section{TEST RESULTS \\ Volumetric response}

Figure 2 shows the volumetric response of the specimens subject to $1 \mathrm{D}$ compression. The initial void ratio of the virgin specimen in each loading series was made essentially the same $(1 \cdot 302 \pm 0 \cdot 009)$. The specimens were prepared carefully and measurement of their dimensions and weight was carried out with high precision. It is believed that the evaluated void ratio should be very accurate. Therefore, whether the normal compression lines (NCLs) converge or not with increasing stress could be judged with high confidence. The following observations can be made.

- The 'yield stress' (indicated by the arrows in Fig. 2) of the virgin specimens falls between 1 and $3 \mathrm{MPa}$, which is much lower than the peak compressive strength (of the order of dozens of megapascal) concluded from single grain crushing tests on more than 30 particles of the same size range. Similar observations were made by Nakata et al. (1999, 2001b).

- The initial void ratio decreases with an increasing number of reconstitutions despite attempts to prepare each reconstituted specimen as loosely as possible. It is anticipated that the smaller sized crushed grains produced during each compression test (see also Fig. 3) fill the voids among larger particles and thus facilitate the formation of a denser assemblage.

- With an increasing number of reconstitutions (producing a more well-graded assemblage) the 'yield stress' becomes less distinctive.

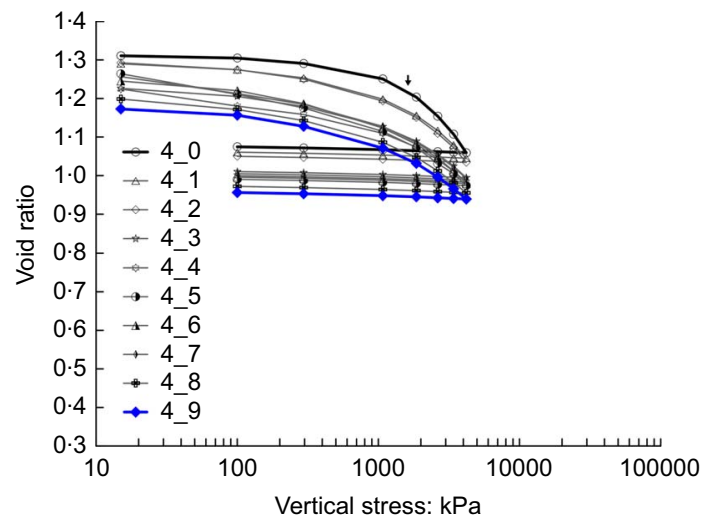

(a)

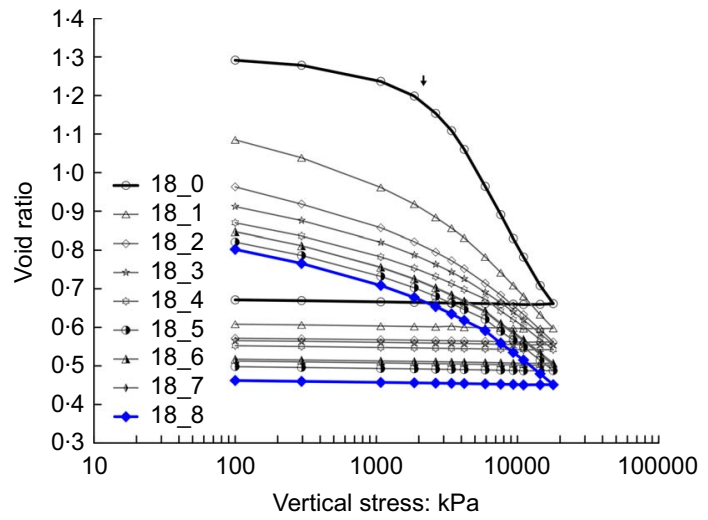

(c)

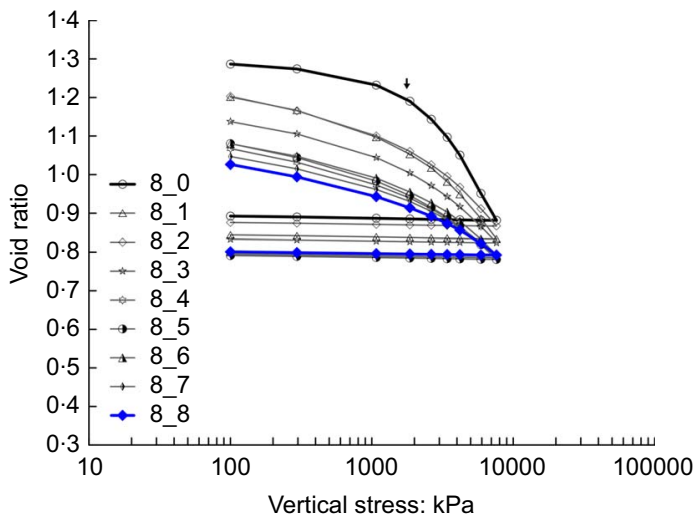

(b)

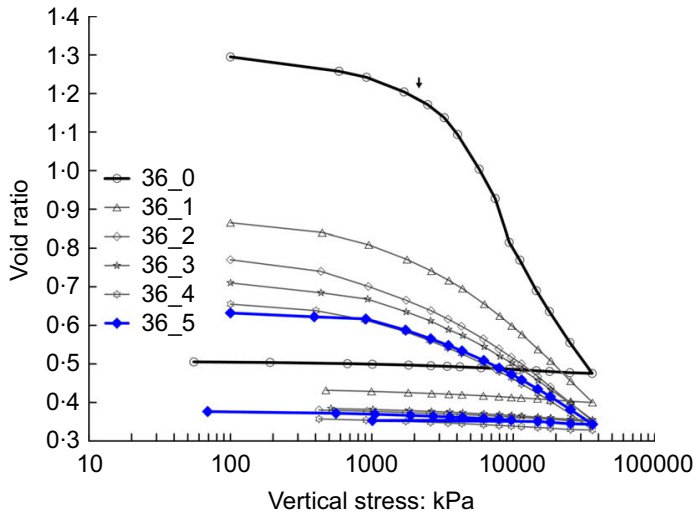

(d)

Fig. 2. One-dimensional compression curves for different test series: (a) $4 \mathrm{MPa}$; (b) $8 \mathrm{MPa}$; (c) $18 \mathrm{MPa}$; (d) $36 \mathrm{MPa}$ 


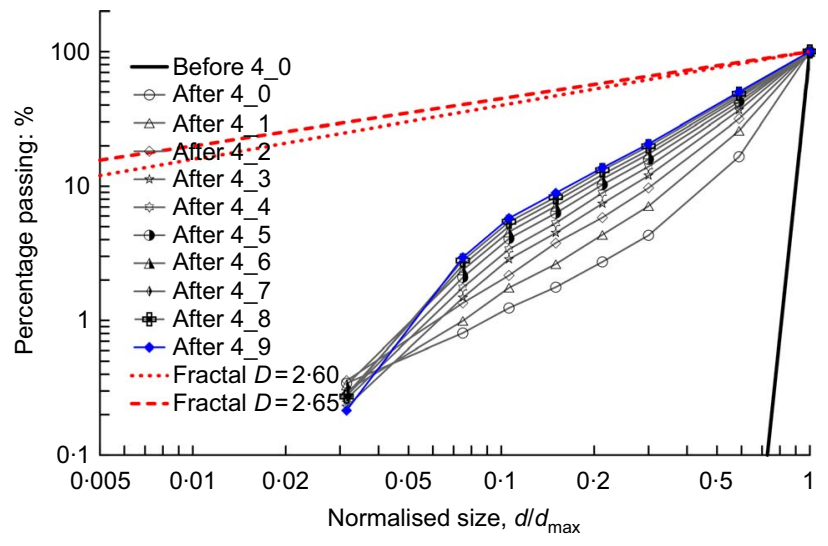

(a)

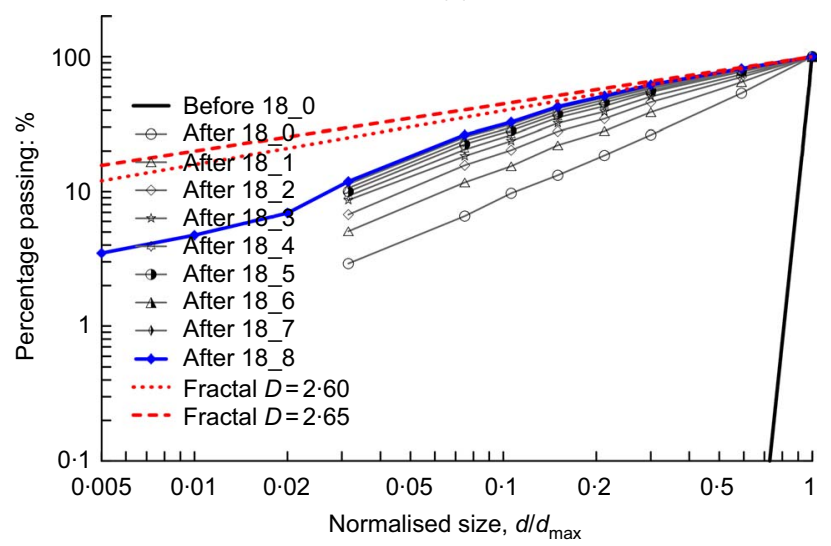

(c)

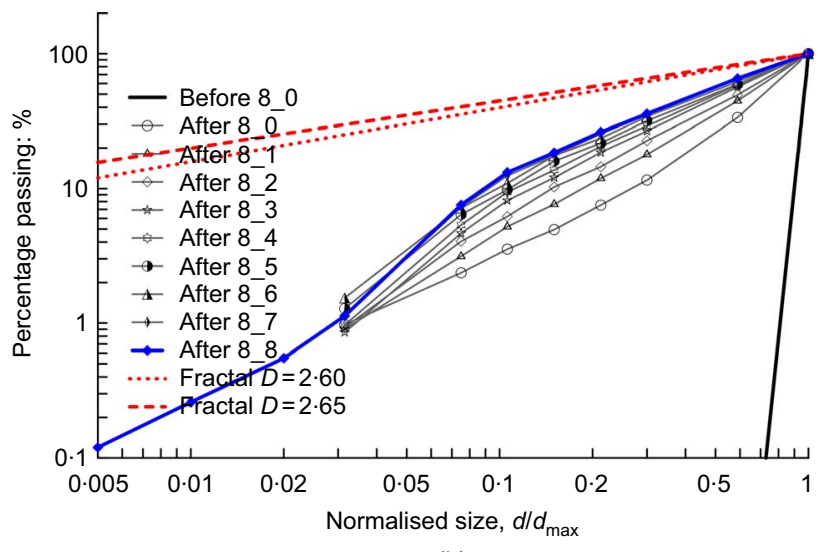

(b)

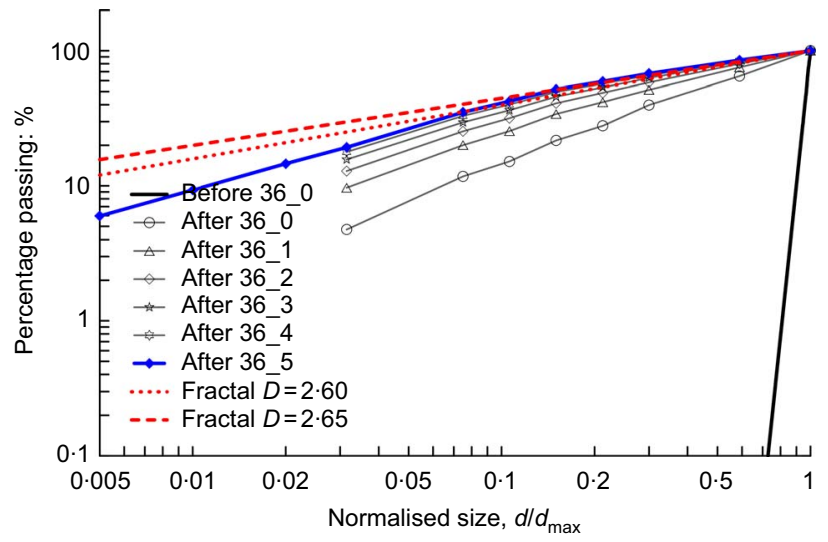

(d)

Fig. 3. Evolution of grain grading during compression: (a) $4 \mathrm{MPa}$; (b) $8 \mathrm{MPa}$; (c) $18 \mathrm{MPa}$; (d) $36 \mathrm{MPa}$

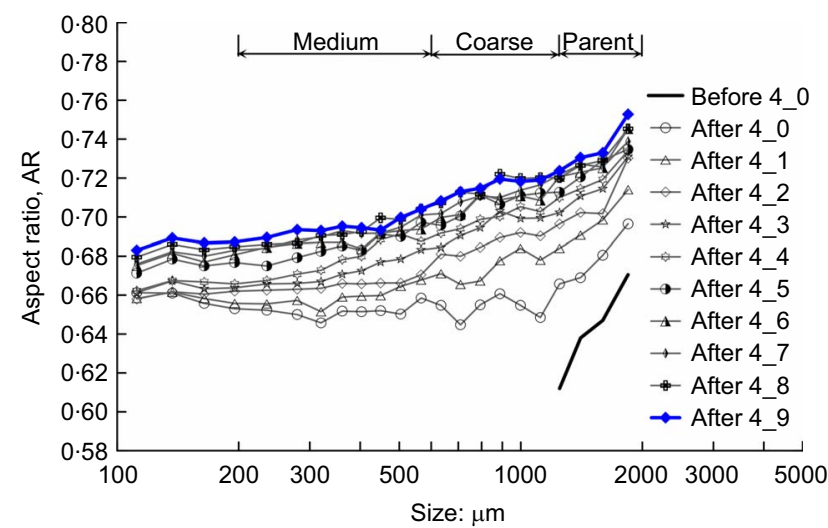

(a)

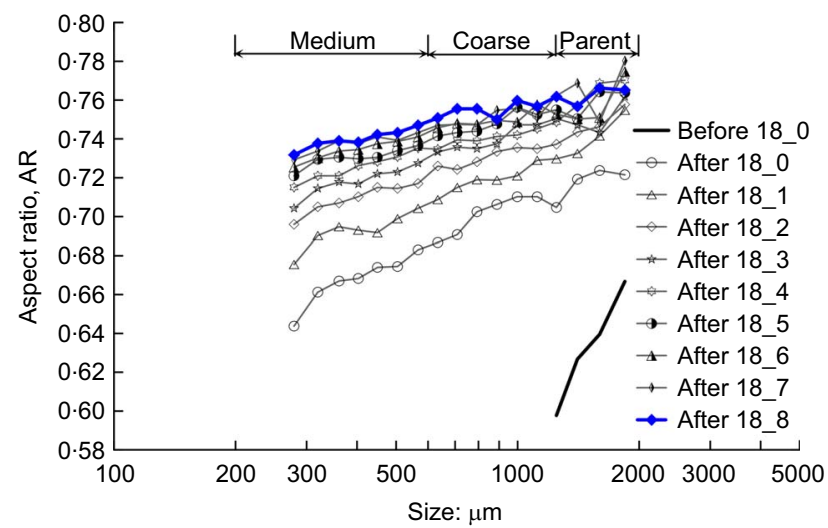

(c)

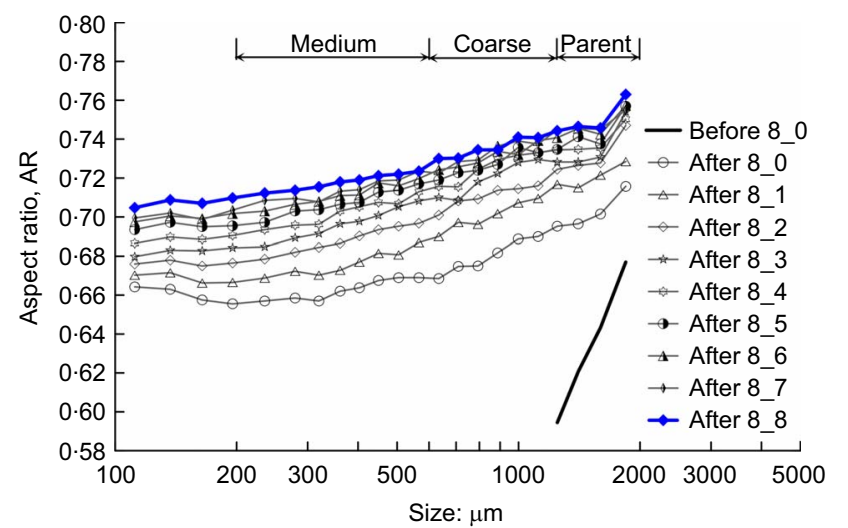

(b)

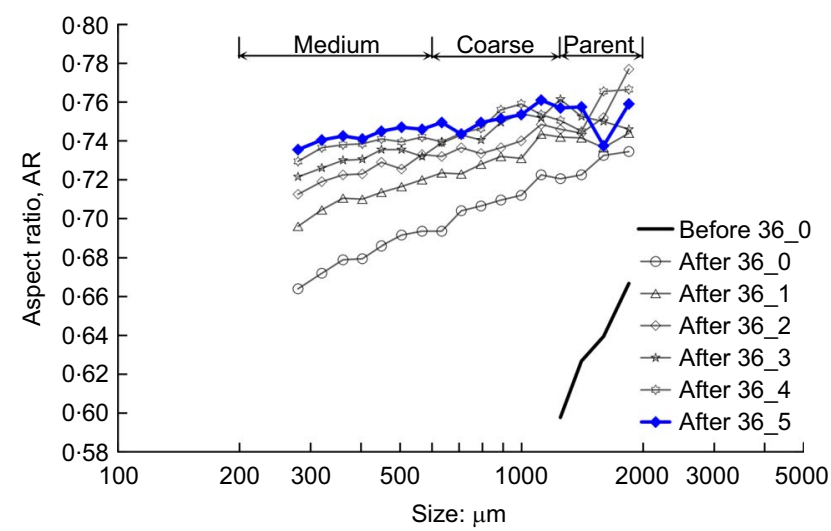

(d)

Fig. 4. Evolution of aspect ratio of grains during compression: (a) $4 \mathrm{MPa}$; (b) $8 \mathrm{MPa}$; (c) $18 \mathrm{MPa}$; (d) $36 \mathrm{MPa}$ 


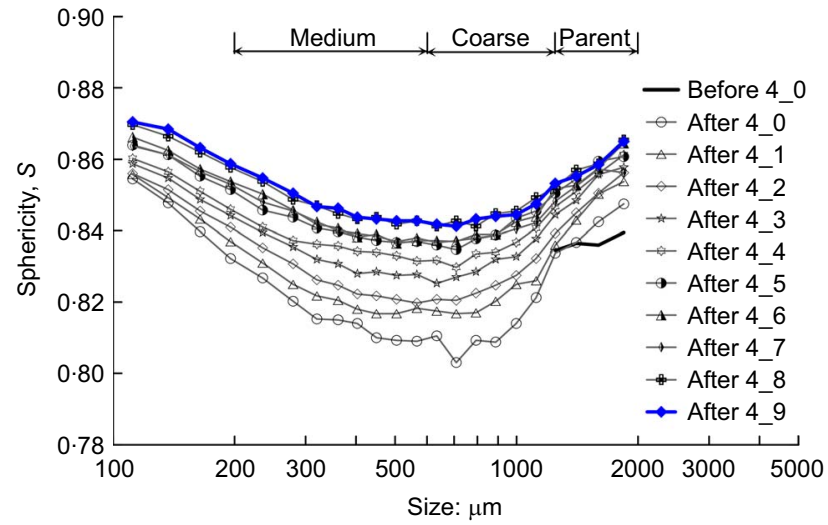

(a)

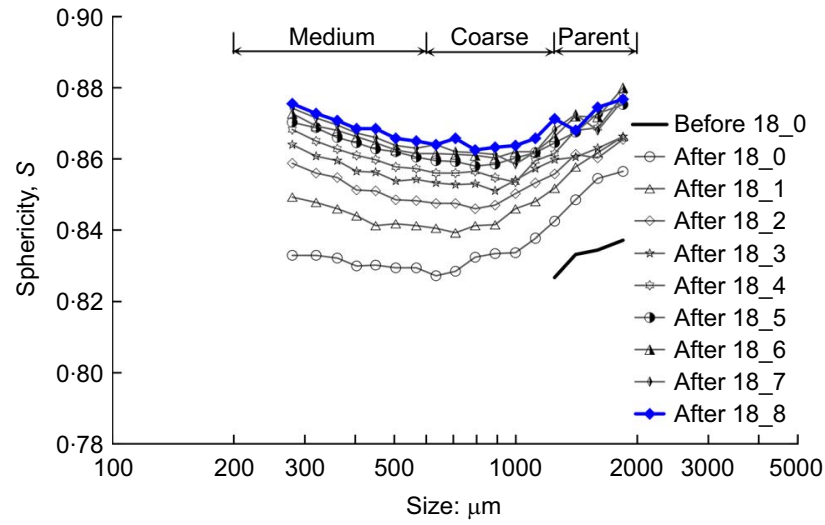

(c)

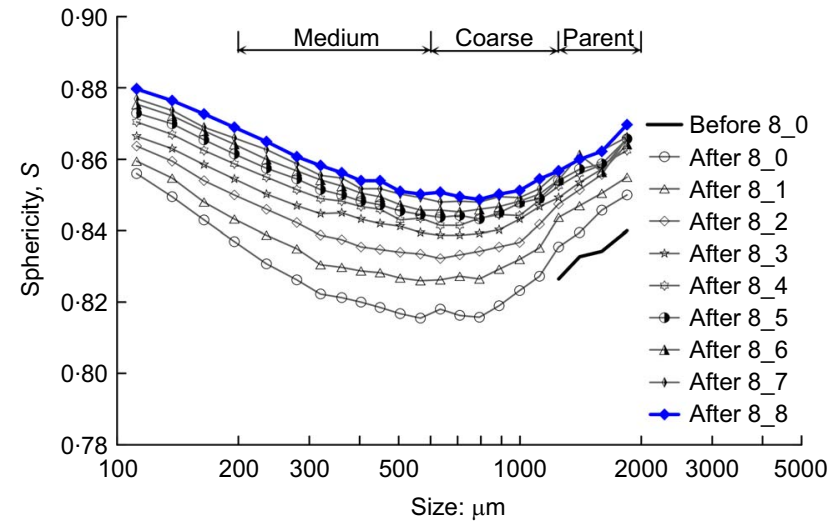

(b)

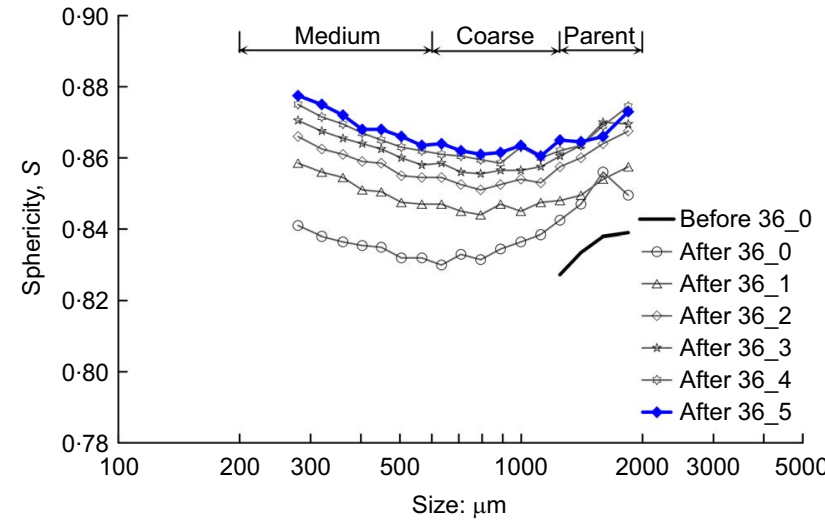

(d)

Fig. 5. Evolution of sphericity of grains during compression: (a) $4 \mathrm{MPa}$; (b) $8 \mathrm{MPa}$; (c) $18 \mathrm{MPa}$; (d) $36 \mathrm{MPa}$

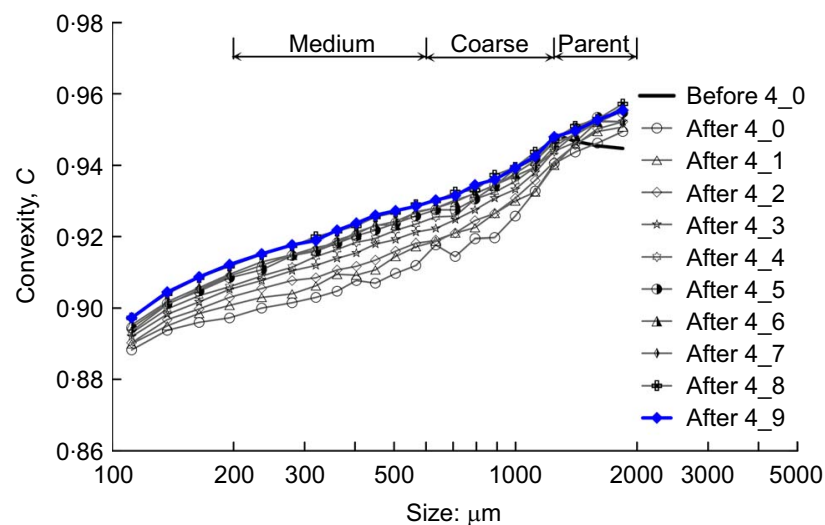

(a)

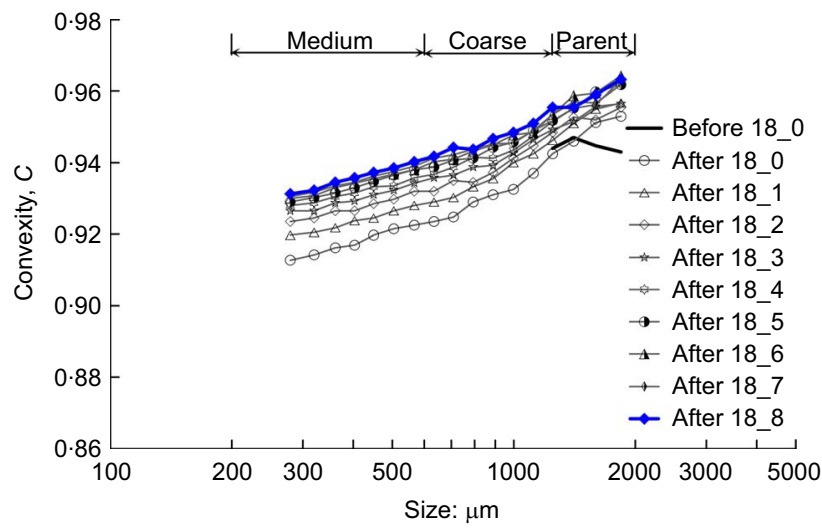

(c)

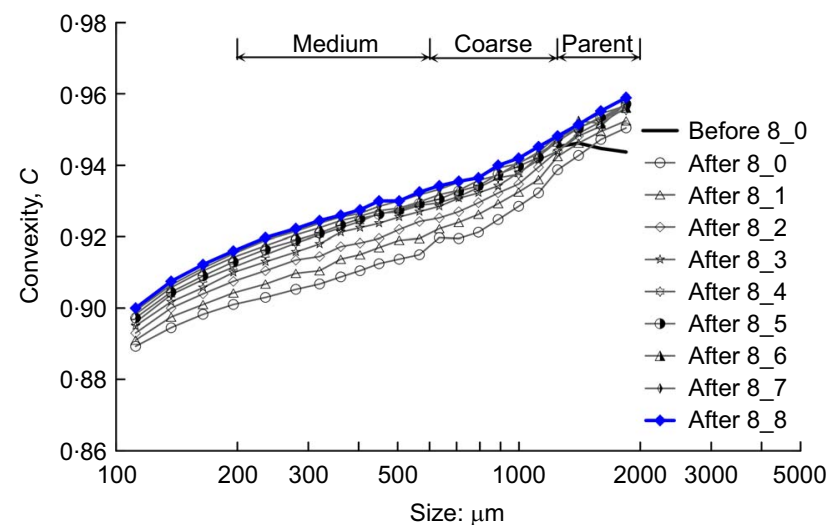

(b)

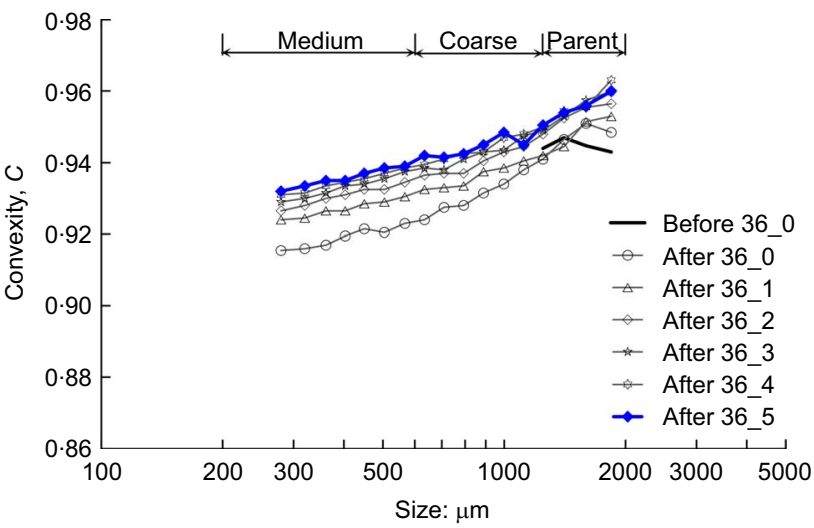

(d)

Fig. 6. Evolution of convexity of grains during compression: (a) $4 \mathrm{MPa}$; (b) $8 \mathrm{MPa}$; (c) $18 \mathrm{MPa}$; (d) $36 \mathrm{MPa}$ 


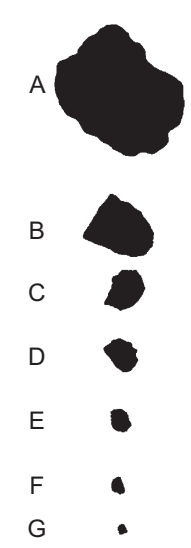

Fig. 7. Images of some randomly selected grains at $36 \mathrm{MPa}$ (final limiting grading)

- At stresses of 4, 8 and $18 \mathrm{MPa}$, the NCLs have not yet converged with an increasing number of reconstitutions; despite that, a rather stable grading is observed in each case (Fig. 3). Nevertheless, at $36 \mathrm{MPa}$ stress, a unique NCL can be observed after a few remouldings (comparing tests 36_4 and 36_5). In addition to the elevated stresses, reconstitution - which facilitates grain breakage - appears to be essential in order to yield a unique NCL.

\section{Evolution of grading}

Figure 3 shows the grain grading at different stages of each loading series. Grading of grains smaller than $63 \mathrm{~m}$ (aperture limit of the mechanical sieves) was not quantified except for the last specimen of a loading series where hydrometer analysis was undertaken to include the grading distribution of the fines. The change of grain grading before and after the first compression is noticeable. The amount of this virgin breakage increases with increasing vertical stresses. The change in grading reduces gradually with an increasing number of reconstitutions and becomes negligible when the number of reconstitutions becomes large. In other words, the production of crushed grains by 1D compression slows down with repeated reconstitutions. For each overburden stress, a stable limiting grading can be obtained (the line annotated with diamond symbols). The coarse-grain segment $\left(d / d_{\max }>0 \cdot 1\right)$ of the stable limiting grading can be described by a power law (linear curve in the double-logarithm space) with a corresponding fractal dimension (equal to three minus the curve gradient) of $1 \cdot 73,2 \cdot 10,2 \cdot 48$ and 2.60 for the case of $4,8,18$ and $36 \mathrm{MPa}$ respectively. For reference, the gradings using fractal dimensions of 2.60 and 2.65 are also shown in Fig. 3 (McDowell \& Bolton, 1998; Einav, 2007). It can be seen that the limiting grading comes close to the grading with $D=2.65$ only when the stress becomes large. Comparing the series of 18 and $36 \mathrm{MPa}$ loadings, it is anticipated that a further increase in the overburden stress facilitates the

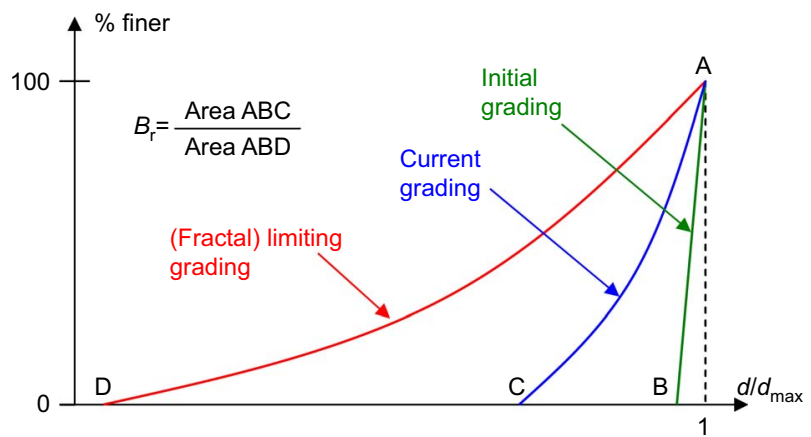

Fig. 8. Definition of relative breakage $B_{\mathrm{r}}$

production of finer grains, which makes the limiting grading fit better by a linear curve in the double-logarithm space. The results imply that, for $1 \mathrm{D}$ compressions, both elevated stress and reconstitution are essential to produce a stable grading with a fractal dimension close to $2 \cdot 65$. This is in contrast to the shear tests in which the ultimate grading can be achieved by high shear strains at relatively low applied stress (Coop et al., 2004).

\section{Evolution of grain shape}

Figures 4-6 show the evolution of grain shape parameters. A perfect sphere gives all parameters equal to unity. As shown in Fig. 4, the parent grains are comparatively elongated (with AR in the range 0.60-0.68). After the first compression, a noticeable increase in AR can be seen. Such an increase becomes more obvious when higher overburden stresses are applied. Furthermore, with an increasing number of reconstitutions the AR increases gradually and, similar to the grading curve, it evolves at a decreasing rate. Considering Fig. 4 and Fig. 5 together, apparently higher vertical stresses could produce more-rounded crushed grains (i.e. an increase in AR and $S$ ). The resulting grains are elliptical. Figure 6 reveals grain compactness at different stages of the tests. In general, the grain convexity (for both parent and crushed grains) is quite high (close to 1). Furthermore, convexity decreases slightly with decreasing grain size, and the same trend was reported by Altuhafi \& Coop (2011). It is anticipated that larger grains are more favourable to surface abrasion, which should increase grain compactness. In general, the ranges of all three characteristic parameters for different size particles reduce with an increasing number of reconstitutions. In other words, self-similar grains at the limiting grading were obtained. Figure 7 shows QicPic images of some randomly selected grains from the final specimen at $36 \mathrm{MPa}$ compression. Again, as shown in the corresponding data in Table 1, grains of different sizes show their grain characteristic parameters varying over a narrow range.

Following Einav (2007), a breakage parameter called relative breakage $\left(B_{\mathrm{r}}\right)$ is defined by the ratio of area $\mathrm{ABC}$ to

Table 1. Characteristic parameters of randomly selected grains shown in Fig. 7

\begin{tabular}{l|c|c|c}
\hline Image & Feret minimum: $\mu \mathrm{m}$ & Aspect ratio & Sphericity \\
\hline A & $1557 \cdot 5$ & $0 \cdot 749$ & $0 \cdot 870$ \\
B & $815 \cdot 5$ & $0 \cdot 756$ & $0 \cdot 860$ \\
C & $532 \cdot 4$ & $0 \cdot 743$ & $0 \cdot 867$ \\
D & $406 \cdot 7$ & $0 \cdot 752$ & $0 \cdot 880$ \\
E & $291 \cdot 7$ & $0 \cdot 746$ & $0 \cdot 879$ \\
F & $203 \cdot 3$ & $0 \cdot 743$ & $0 \cdot 872$ \\
G & $129 \cdot 3$ & $0 \cdot 756$ & $0 \cdot 871$ \\
\hline
\end{tabular}




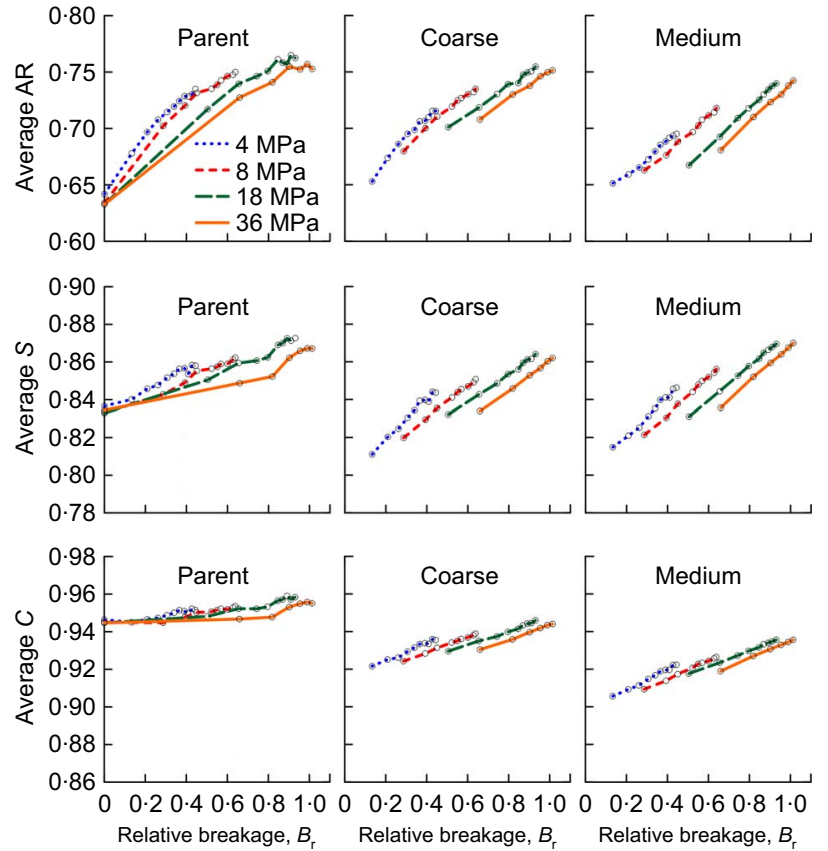

Fig. 9. Effect of loading stress on the evolution of shape parameters against $B_{\mathrm{r}}$

area $\mathrm{ABD}$, as shown in Fig. 8 where the grain size is normalised by the maximum grain size. $B_{\mathrm{r}}$ represents the relative position of a grading curve with respect to the initial grading $\left(B_{\mathrm{r}}=0\right)$ and the ultimate fractal grading $\left(B_{\mathrm{r}}=1\right)$. Figure 9 shows the shape parameters plotted against $B_{\mathrm{r}}$. In this study, the ultimate fractal grading curve was evaluated by adopting $D=2 \cdot 65$. The dots in Fig. 9 denote the end of a compression test when QicPic analyses were performed. The grains are divided into three groups according to their size: 'parent' - indicating the size of the parent grains $(1 \cdot 18-2.00 \mathrm{~mm})$; 'coarse' $(0 \cdot 60-1 \cdot 18 \mathrm{~mm})$;

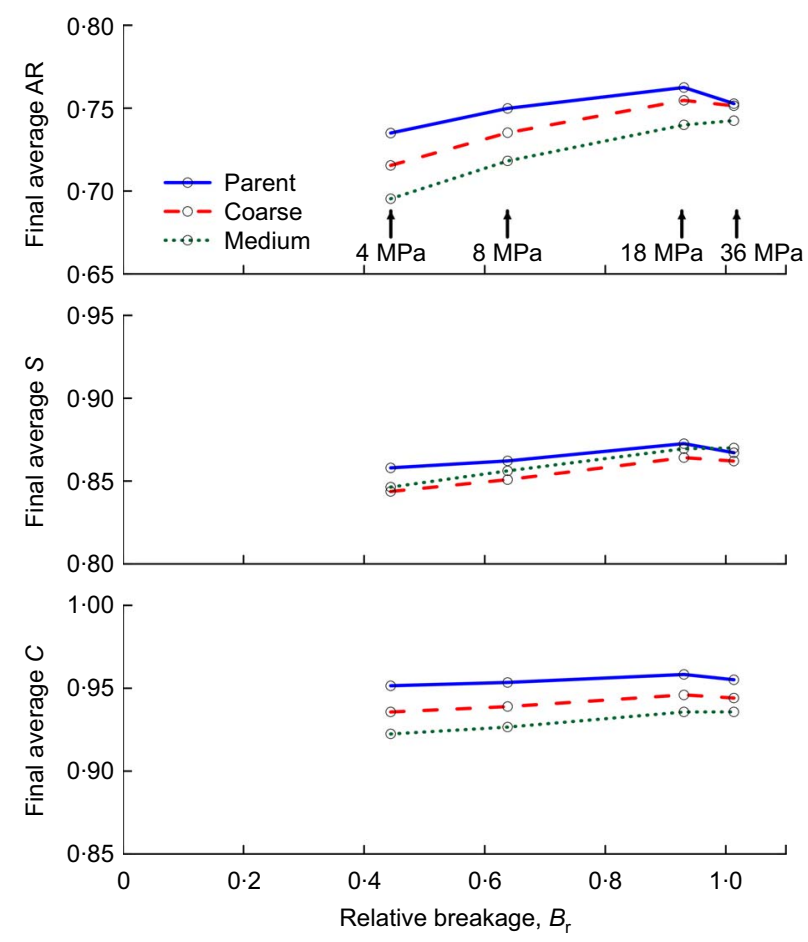

Fig. 10. Variation of shape parameters with $B_{r}$

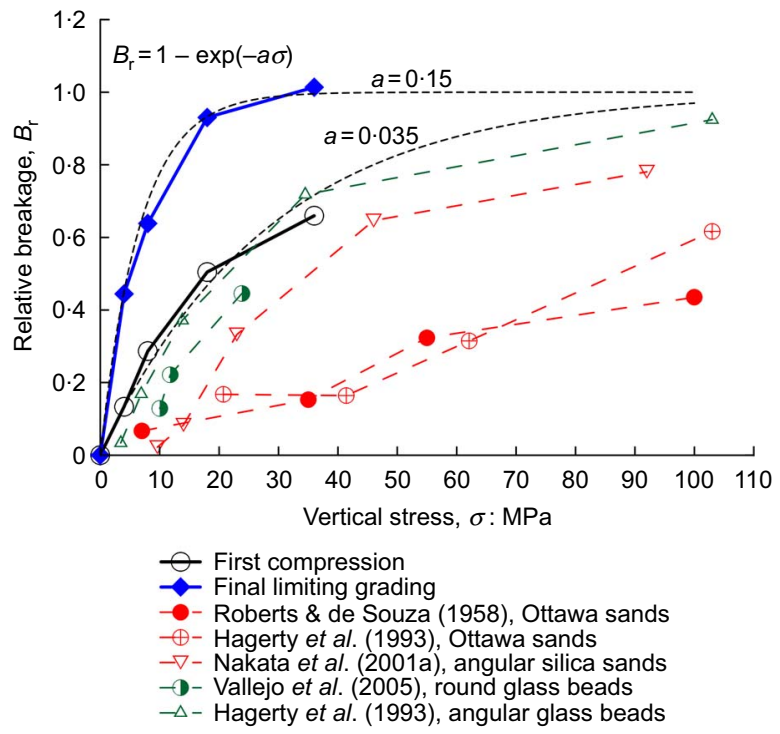

Fig. 11. Influence of materials hardness and shape on the development of $B_{\mathrm{r}}$ with stress

and 'medium' $(0 \cdot 20-0 \cdot 60 \mathrm{~mm}) . B_{\mathrm{r}}$ increases with the number of reconstitutions but cannot achieve unity when the overburden stress is low. For the parent group, the relationship between each average grain parameter and $B_{\mathrm{r}}$ is not unique. The average grain characteristic parameters (especially $\mathrm{AR}$ and $S$ ) increases with $B_{\mathrm{r}}$ at a lower rate as stress increases. For both the coarse and medium groups, again a non-unique relationship between the shape parameters and $B_{\mathrm{r}}$ can be seen. As stress increases, the curves consistently shift to the right with essentially the same gradient (except AR and $S$ of the first few reconstituted specimens at $4 \mathrm{MPa}$ of the coarse group). The grain characteristics parameter at $B_{\mathrm{r}}=0$ is undefined for these two groups since the initial grading contains no grains in these two size ranges.

The grain parameters associated with the final limiting grading in each loading case are plotted against $B_{\mathrm{r}}$ in Fig. 10. The change of shape parameters becomes negligible when $B_{\mathrm{r}}$ is close to unity. Furthermore, the shape parameters of grains in different size groups are very close to each other when $B_{\mathrm{r}}$ approaches unity. This indicates that not only the grading but also the grain shape becomes rather stable when the grading is close to the final limiting fractal one. In other words, the assemblages become fractal (i.e. grains with self-similarity).

\section{Crushability}

Figure 11 shows the variation of $B_{\mathrm{r}}$ (after the first compression and at the final limiting grading) with stress. $B_{\mathrm{r}}$ increases gradually with stress and the trend can be wellcaptured by an inverse-exponential law with the factor $a$ indicating the rate of increase. $B_{\mathrm{r}}$ derived from the final limiting grading increases faster than that of the first compression due to the effect of repeated remoulding, which facilitates crushing. Figure 11 also includes 1D compression data from other studies on sands and glass beads (Roberts \& de Souza, 1958; Hagerty et al., 1993; Nakata et al., 2001a; Vallejo et al., 2005). The rounded Ottawa sands show a noticeably slower change in $B_{\mathrm{r}}$ with stress as compared to the round glass beads and the studied calcareous sands. This is probably due to their difference in material compressive strength. Angularity also makes the grains more vulnerable to breakage: the curves of the 
angular glass beads are therefore located higher (more breakage at the same stress) than the round glass beads.

\section{CONCLUSIONS}

This study presents the effects of 1D overburden stress and remoulding on the evolution of grading and grain shape. The following conclusions are drawn.

- A limiting grading can be obtained by repeated remoulding and loading.

- The evolution of grain shape with remoulding depends on the magnitude of the applied stress. However, different size grains having very similar shape characteristics (selfsimilarity) are obtained at the final limiting grading.

- The final limiting grading comes close to the ultimate fractal one (with $D=2 \cdot 65$ ) only when high stress compression is performed on repeatedly remoulded samples subject to $1 \mathrm{D}$ compression.

\section{ACKNOWLEDGEMENTS}

The QicPic optical analyser was funded by the University Development Fund at the University of Hong Kong. The current study was financially supported by the Seed Funding Programme for Basic Research at the University of Hong Kong (project no. 201211159157).

\section{REFERENCES}

Altuhafi, F. N. \& Coop, M. R. (2011). Changes to particle characteristics associated with the compression of sands. Géotechnique 61, No. 6, 459-471.

Coop, M. R., Sorensen, K. K., Bodas Freitas, T. \& Georgoutsos, G. (2004). Particle breakage during shearing of a carbonate sand. Géotechnique 54, No. 3, 157-163.

Einav, I. (2007). Breakage mechanics - part I: theory. J. Mech. Phys. Solids 55, No. 6, 1274-1297.
Hagerty, M. M., Hite, D. R., Ullrich, C. R. \& Hagerty, D. J. (1993). One-dimensional high-pressure compression of granular media. J. Geotech. Engng ASCE 119, No. 1, $1-18$.

Lade, P. V. \& Yamamuro, J. A. (1996). Undrained sand behaviour in axisymmetric tests at high pressures. J. Geotech. Engng ASCE 122, No. 2, 120-129.

Leung, C. F., Lee, F. H. \& Yet, N. S. (1996). The role of particle breakage in pile creep in sand. Can. Geotech. J. 33, No. 6, 888898.

McDowell, G. R. \& Bolton, M. D. (1998). On the micromechanics of crushable aggregates. Géotechnique 48, No. 5, 667-679.

McDowell, G. R., Bolton, M. D. \& Robertson, D. (1996). The fractal crushing of granular materials. J. Mech. Phys. Solids 44, No. 12, 2079-2102.

Miao, G. \& Airey, D. (2013). Breakage and ultimate states for a carbonate sand. Géotechnique 63, No. 14, 1221-1229.

Nakata, Y., Hyde, A. F. L., Hyodo, M. \& Murata, H. (1999). A probabilistic approach to sand particle crushing in the triaxial test. Géotechnique 49, No. 5, 567-583.

Nakata, Y., Hyodo, M., Hyde, A. F. L., Kato, Y. \& Murata, H. (2001a). Microscopic particle crushing of sand subjected to high pressure one-dimensional compression. Soils and Found. 41, No. 1, 69-82.

Nakata, Y., Kato, Y., Hyodo, M., Hyde, A. F. L. \& Murata, H. (2001b). One-dimensional compression behaviour of uniformly graded sand related to single particle crushing strength. Soils and Found. 41, No. 2, 39-51.

Roberts, J. E. \& de Souza, J. M. (1958). The compressibility of sands. Proc. Am. Soc. Test. Mater. 58, 1269-1277.

Vallejo, L. E., Lobo-Guerrero, S. \& Chik, Z. (2005). A network of fractal force chains and their effect in granular materials under compression. In Fractals in engineering: new trends in theory and applications (Levy-Vehel, J. \& Lutton, E. (eds)). London: Springer, pp. 67-80.

Yasufuku, N. \& Hyde, A. F. L. (1995). Pile end-bearing capacity in crushable sands. Géotechnique 45, No. 4, 663-676.

Zhang, X. \& Baudet, B. A. (2013). Particle breakage in gap-graded soil. Géotech. Lett. 3, No. 2, 72-77. 